\title{
TITLE:
}

\section{Remote control of apomorphine infusion rate in Parkinson's disease: Real-time dose variations according to the patients' motor state. A proof of concept}

\author{
Rodriguez-Molinero $\mathrm{A}^{1,2}$, Pérez-Martínez DA ${ }^{3}$, Gálvez $\mathrm{C}^{1}$, Hernández-Vara $\mathrm{J}^{4}$, \\ Martinez-Castrillo $\mathrm{JC}^{5}$, Álvarez $\mathrm{R}^{6}$, de Fabregues $\mathrm{O}^{4}$, Samà $\mathrm{A}^{7}$, Pérez-López $\mathrm{C}^{7}$, \\ Romagosa $\mathrm{J}^{7}$, Bergman $\mathrm{Y}^{8^{*}}$
}

${ }^{1}$ Clinical Research Unit, Fundación Sant Antoni Abat (Consorci Sanitiari del Garraf), Barcelona, Spain

${ }^{2}$ School of Engineering and Informatics, National University of Ireland at Galway, Galway, Ireland.

${ }^{3}$ Department of Neurology, Hospital Universitario Infanta Cristina, Parla-Madrid, Spain.

${ }^{4}$ Department of Neurology, Hospital Universitari Vall d'Hebron, Barcelona, Spain.

${ }^{5}$ Department of Neurology, Hospital Universitario Ramón y Cajal, Madrid, Spain.

${ }^{6}$ Department of Neurology, Hospital Universitari Germans Trias i Pujol, Barcelona, Spain.

${ }^{7}$ Technical Research Centre for Dependency Care and Autonomous Living, Universitat Politècnica de Catalunya, Barcelona, Spain

${ }^{8}$ Maccabi Institute for Health Services Research, Tel-Aviv, Israel.

* Author deceased.

*Corresponding Author:

Alejandro Rodríguez Molinero. $\mathrm{MD} / \mathrm{PhD}$

C/ Sant Josep 21-23, Vilanova i la Geltrú, 08800 Barcelona

Tel: 34-649408331 Fax: 34-938140042

rodriguez.molinero@gmail.com 


\section{KEYWORDS}

Parkinson; Apomorphine; Telehealth 


\section{INTRODUCTION}

Patients with advanced Parkinson's disease experience motor fluctuations that seem to be related with variations in their serum levels of L-dopa and dopaminergic agonists. Therefore, drug infusion pumps have been developed with the aim of maintaining stable serum levels and reducing motor fluctuations [1]. Such pumps are usually programmed with a constant infusion rate. Some pumps also allow the patient to self-administer a rescue dose when necessary. Adjusting the schedule for a patient often requires several visits to the physician or admissions to hospital, especially at the beginning of the treatment [2].

Information and communication technologies make it possible to control infusion pumps remotely, thus reducing the number of necessary visits to the physician and/or improving the control of the disease. Furthermore, the technological development of sensors allows for the design of smaller and more wearable systems to detect motor fluctuations [3]. Such sensors can automatically detect the moments in which a patient needs more doses [4], thus allowing for automatic or semi-automatic control of the infusion pump. Consequently, the infusion rate might be adjusted to the unpredictable motor fluctuations of some patients in real time.

During the course of the HELP project, a technological system for remote real-time control of apomorphine pumps was developed [5]. The system includes a kinematic sensor able to detect motor symptoms and send the information through a mobile phone (gateway) to a central server. In the server, the symptoms can be analyzed by an expert, who readjusts the apomorphine dose accordingly with the detected motor phase (ON or OFF). The new dose is sent back through the mobile-gateway to the wireless pump.

In this article, we report the results of a proof of concept, where real-time dose variations were introduced according to the patient's motor state. Although the HELP system includes an inertial sensor that can detect the patient's motor state, in this proof of concept, dose decisions were not based on the readings from the sensor (in process of validation) but on the motor state the patients reported by telephone. 


\section{METHOD}

Quasi-experimental study conducted on a group of 6 patients, with a before-after design (pre-post intervention). All patients included suffered from idiopathic Parkinson's disease, according to the criteria of the Brain Bank of London [6]; they experienced motor fluctuations and used apomorphine pumps. Patients with severe dyskinesia or dystonia, severe orthostatic hypotension, risk of Torsades-de-Pointes arrhythmia, anaemia, liver or kidney failure and those wearing other implantable electronic devices were excluded. Participants were selected by convenience sampling in 4 hospitals. The field work was conducted in Madrid and Barcelona (Spain) between October 2012 and March 2013.

Participants - who were already users of apomorphine pump - used the HELP infusion pump programmed to a constant rate corresponding to the patient's habitual dose, for 56 days (pre-intervention phase). Subsequently, they used the same pump for 5-6 days, though with remotely controlled variations in the infusion rate depending on their motor state (post-intervention phase). The change-of-dose protocol involved using the patients' habitual doses when they were in the On state and $25 \%$ or $50 \%$ higher rates (depending on their tolerance as evaluated in pre-test trials) when they entered the Off phase and until they returned to the On phase (when previous basal rate was set again). New doses were set in the pump from a remote position, using the specific software and communications of the HELP system. Patients could report changes in their motor activity by calling at any time a medical operator, who was in charge of re-adjusting the dose. The medical operator also called the patients every two hours.

The main study variable was the time patients spent in either of the states (On, Off or a "intermediate" phase between both) during the continuous infusion period (preintervention phase) and the remotely controlled infusion period (post-intervention phase). Patients were asked to record their motor state every 30 minutes on a specially designed paper calendar. The medical operator who was in charge of calling them by telephone every 2 hours also recorded the patients' motor state, thus supplementing the data recorded by patients. For patients using apomorphine rescue, the number of injections, dose and time were recorded. No further modifications were allowed in 
dopaminergic medication during the time of the study. Adverse effects occurred during the experimental time were systematically recorded.

Descriptive statistical analysis was performed for each patient; the total time a patient spent in either of the three above defined motor states was computed. For patients using rescue injections, the number of injections they needed during the time of the study was recorded. The analysis compared all the "post-intervention" time, where apomorphine doses were remotely controlled (6 daily hours minimum), against an equivalent period of the "pre-intervention" phase (same times of the day), where apomorphine doses remained stable at patient's usual rate. Once a patient was included in the analysis, occasions where the dose-change protocol could not be completed due to technical failure were not excluded from the analysis (to avoid a selection bias of ON episodes). Differences in the time-in-Off and the number of necessary apomorphine rescues between the pre- and the post-intervention phases were analyzed by using the Wilcoxon test. Reported adverse effects were recorded.

The study protocol was approved by the Consorci Sanitari del Maresma Ethical Committee. All participants provided informed consent prior to their inclussion in the study. 


\section{RESULTS}

Only 3 participants out of the 6 initially enrolled ones were included in the analysis. Two patients were excluded due to coverage problems that affected remote control of the pump through the HELP system, and one patient was excluded because the remote control system broke down.

Table 1 shows the general characteristics, baseline and increased apomorphine doses and number of days in the study corresponding to every participant patient.

For participants 4 and 6, who did not use apomorphine rescues, the time in On was slightly longer $(7.1 \%, \mathrm{p}=0.17)$ and the time in Off was $10 \%$ shorter $(\mathrm{p}<0.05)$ in the post-intervention phase. Patient 2 used apomorphine rescues during both pre and postintervention phases. Therefore the results in terms of On/Off times were confusing; during the experimental time this patient spent longer times in the On and Off states, and consequently shorter time in the intermediate state, as compared with the first week. However, this patient needed a mean of 4 apomorphine rescues per day during the preintervention phase and 3 rescues per day during the post-intervention period (one third of the latter rescues were administered out of experimental hours). Table 2 shows the results corresponding to these three patients.

Regarding adverse side effects, three cases of low blood pressure were recorded, two of them during the pre-intervention period. The three cases were self-limited and no intervention was required. One of the patients experienced excessive somnolence during the post-intervention period, which could be related with variations in the apomorphine infusion rate. No further adverse side effects with a possible relationship with the treatment were recorded. 


\section{DISCUSSION}

The present study is a proof of concept, designed to test remote control of apomorphine infusion pumps. Furthermore, the possible effects on the patient of an intensive realtime dose adjustment that depends on the patient's motor state, have been explored. As far as we know this is the first time that either of these challenges are attempted.

Remote control of the apomorphine pump was acceptable for 3 out of 6 patients, which demonstrates that such monitoring method is technically feasible. However, the fact that the HELP system depends on the mobile phone network prevents its use in Spain, where many geographical locations have a deficient mobile data broadband coverage. Remote control could potentially facilitate initial and long-term dose adjustment, thus reducing the number of admissions to hospital and visits to the physician needed for dose adjustment, especially during the initial phases of treatment [2].

Intensive adjustment of the apomorphine dose, in response to patients' motor fluctuations, could be done either manually by the patient or automatically through the use of new technologies for detection of motor symptoms [3]. This study is a proof of concept which, despite limited results due to the small sample size, shows a tendency towards an improved symptom control (shorter time in Off; less rescues needed) during the intervention periods, where the dose was changed depending on the patient's motor state. These results support the need for further studies aimed at exploring the possible benefits of this alternative dosing method.

Although this study is only an initial proof of concept, we postulate that "intelligent" devices that detect the patient's motor state and act accordingly, are feasible and constitute a line of research with a great potential for the future. The increasing development of mobile data broadband in our country will facilitate the use of such tools for telemedicine projects like the present one. 


\section{ACKNOWLEDGMENTS}

In memoriam of Dr. Yossi Bergman, who contributed enormously to the HELP Project with his scientific knowledge, kind manners and sustained enthusiasm.

We would like to thank Dr. Antonio Yuste Marco, Ms. Esther Valldosera Dorado and Ms. Natalia Gonzalo León for the work done in research management and administration.

\section{COMPETING INTERESTS}

The authors have no commercial associations or sources of support that might pose a conflict of interest.

\section{FUNDING}

This study was supported by the HELP Project (Home-based Empowered Living for Parkinson's disease Patients - AAL-2008-1-022), funded by European commission, and the Spanish Ministerio de Industria, Energía y Turismo within the Ambient Assisted Living Joint Programme. 


\section{BIBLIOGRAPHY}

[1] Volkmann J, Albanese A, Antonini A, et al. Selecting deep brain stimulation or infusion therapies in advanced Parkinson's disease: an evidence-based review. J Neurol. 2013;11: 2701-14.

[2] Castaño B, Mateo D,Giménez-Roldán S. Introducción ambulatoria de apomorfina en infusión subcutánea continua en pacientes con enfermedad de Parkinson avanzada: experiencia y recomendaciones Neurología 2007; 22(3):133-137

[3] Rodríguez-Martín D, Pérez-López C, Samà A et al. A wearable inertial measurement unit for long-term monitoring in the dependency care area. Sensors (Basel) 2013; 10:14079-104.

[4] Rodríguez-Molinero A, Samà A, Pérez-Martínez DA. Validation of a portable device for mapping motor and gait disturbances in Parkinson's disease. JMIR mHealth uHealth (forthcoming) doi:10.2196/mhealth.3321 (NOTE TO REVIEWERS: doi still not working)

[5] Ahlrichs, C., Samà, A., Simon, J. R., Herrlich, S., Rodrıguez-Molinero, A. Help: Optimizing treatment of parkinson's disease patients. In 3rd International Conference on the Elderly and New Technologies, Castellón, Spain. 2012

[6] Hughes AJ, Daniel SE, Kilford L et al. Accuracy of clinical diagnosis of idiopathic Parkinson's disease: a clinico-pathological study of 100 cases. J Neurol Neurosurg Psychiatry; 1992; 55:181-4. 